\title{
Investigation of acoustic fields in the arctic zone with uneven ice cover
}

\author{
Vladimir Korochentsev ${ }^{1, *}$, Chen Wenjian ${ }^{2}$, Victor Petrosyants ${ }^{1}$, Tatiana Lobova ${ }^{3}$, Julia \\ Shpak ${ }^{1}$. \\ ${ }^{1}$ FEFU, Polytechnical Institute, Department of Electronics, Telecommunications and Instrumentation, \\ Russia \\ ${ }^{2} \mathrm{HIU}$, College of underwater acoustic engineering, China \\ ${ }^{3}$ MSUN named after Admiral G.I. Nevelskoy, Russia
}

\begin{abstract}
A mathematical model for elastic wave propagation in an ice cover with uneven relief (hummock) has been developed. The theoretical model is based on the application of "directed" Green's functions. We obtained numerical results for different distances between radiating and receiving antennas installed inside the ice layer and in water medium. An information-measuring system was created to investigate elastic acoustic waves along ice surface based on electo-hydraulic generator. Experiments of high-frequency acoustic signal propagation from electro-hydraulic generator in water-ice-air system were carried out. We illustrated the model validity for the investigation of hydroacoustic wave propagation in real ice conditions.
\end{abstract}

\section{Mathematical model}

We consider the following problem. We are to estimate the field of acoustic wave point sources placed in the ice layer of uneven form (Fig. 1). The following medium characteristics are known: ice, water, air densities and phase velocities of acoustic wave propagation in them; power applied to the radiating antenna; signal frequency; mutual arrangement of sources.

As an initial equation we chose the inhomogeneous Helmholtz equation with Dirac deltafunction in the right part [1-3]:

$$
\Delta P+k^{2} P=\delta\left(\theta, \theta_{0}, r, r_{0}\right),
$$

where $k$ - is the wave number;

$\delta\left(\theta, \theta_{0}, r, r_{0}\right)$ - is the Dirac delta function;

$\theta_{0}, r_{0}$ - are source coordinates;

$\theta, r$ - are estimation point coordinates.

*Corresponding author: ga i uzt@mail.ru 


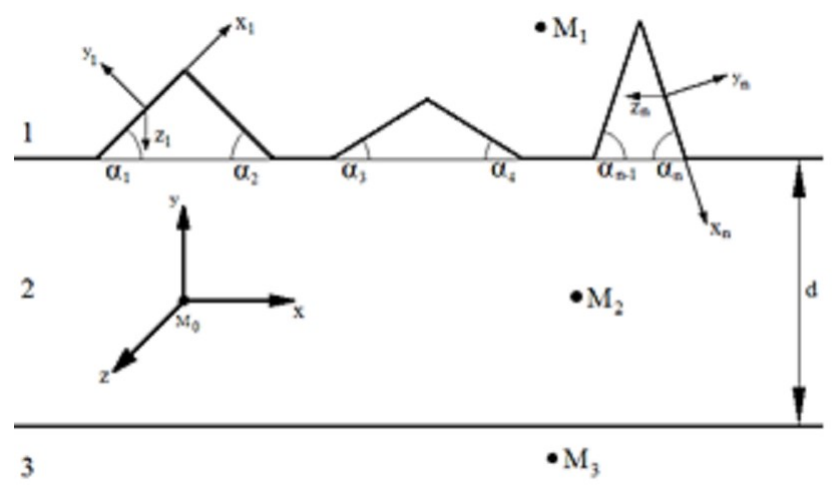

Fig. 1. Geometry of the problem under consideration: $\mathrm{M}_{0}$ - radiation source; 1 - air half-space, 2 - ice layer, 3 - water half-space; $d$ - ice layer thickness.

We introduce generalized angular coordinates for $\varphi=0$ :

$$
\begin{gathered}
U_{1 n}=k_{0} \cdot \sin \left(\alpha \pm \alpha_{n}\right) ; \\
U_{2 n}=k_{0} \cdot \cos \left(\alpha \pm \alpha_{n}\right) ; \\
k_{0}=2 \pi / \lambda .
\end{gathered}
$$

Point M can be located in the air, ice layer or water. To estimate by "directed" Green's functions, we consider the effect of each plane of ice relief on the resulting field. Radiation point source is located at $\mathrm{M}_{0}$ point with the coordinates $x_{0}$ and $y_{0}$. The resulting field in the selected point $\mathrm{M}$ with the coordinates $x$ and $\mathrm{y}$ is equal to the sum of "directed" Green's functions $P_{0}^{n}\left(x_{0 n}, y_{0 n}\right)$, estimated from all the planes of ice relief.

"Directed" Green's function of the waves radiated by a point source is written as

$$
P_{0}^{n}(x)=\frac{i}{2 \pi} \int_{U_{0 \min }^{n}}^{U_{0 \max }^{n}} \frac{F_{0}^{n}\left(U_{0 n}\right)}{\sqrt{k_{0}^{2}-U_{0 n}^{2}}} e^{\left\{i\left(x-x_{0}\right) \sqrt{k^{2}-U_{0 n}^{2}}+\left[y-y_{0}\right] U_{0 n}\right\}} d U_{0 n},
$$

where $F_{0}^{n}\left(U_{0 n}\right)$ is the diagram function:

$$
F_{0}^{n}\left(U_{0 n}\right)=\left\{\begin{array}{c}
1 \text { if } U_{0 \min }^{n} \leq U_{0 n}<U_{0 \max }^{n} \\
0 \text { in other regions }
\end{array}\right.
$$

"Directed" Green's function of the reflected waves has the from

$$
P_{l}^{n}(x)=\frac{i \cdot V}{2 \pi} \int_{U_{l \min }^{n}}^{U_{l \max }^{n}} \frac{F_{l}^{n}\left(U_{l n}\right)}{\sqrt{k_{0}^{2}-U_{l n}^{2}}} e^{\left\{-i\left(x-x_{0}\right) \sqrt{k^{2}-U_{l n}^{2}}+\left[y-y_{0}\right] U_{0 n}\right\}^{2}} d U_{l n},
$$

where $V$ is the interface reflection coefficient determined by the formulas:

$V=\frac{Z_{2}-Z_{1}}{Z_{2}+Z_{1}}-$ for the reflection from upper interface;

$V=\frac{Z_{2}-Z_{3}}{Z_{2}+Z_{3}}$ - for the reflection from lower interface;

$Z_{1}, Z_{2}, Z_{3}-$ air, ice and water impedans.

"Directed" Greens' function for the waves, which transmitted through the interface, is 


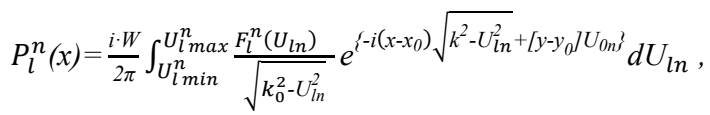

where $W$ is the coefficient of transmission though the interface:

$$
W=1-V .
$$

Resulting field amplitude at $\mathrm{M}$ is determined by the sum of "directed" Green's functions

$$
P_{\Sigma}(x)=\sum_{n=1}^{N}\left[P_{0}^{n}(x)+P_{l}^{n}(x)\right] .
$$

As an example, applying formula (4) we estimate the field from one point source for the frequency of $3 \mathrm{kHz}$ between the radiation point $\mathrm{M}_{0}$ and $\mathrm{M}_{3}$, located in water under the ice layer at the distance up to 100 meters.

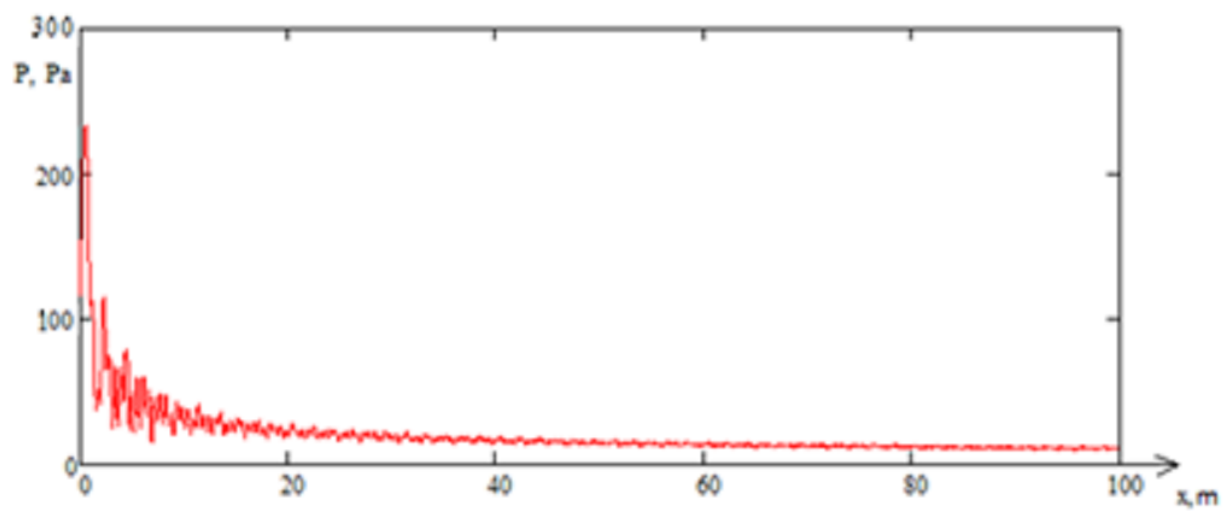

Fig. 2. Distribution of point source field amplitude for the frequency of $3000 \mathrm{~Hz}$.

\section{Experimental equipment}

The experimental equipment is illustrated in Fig. 3.

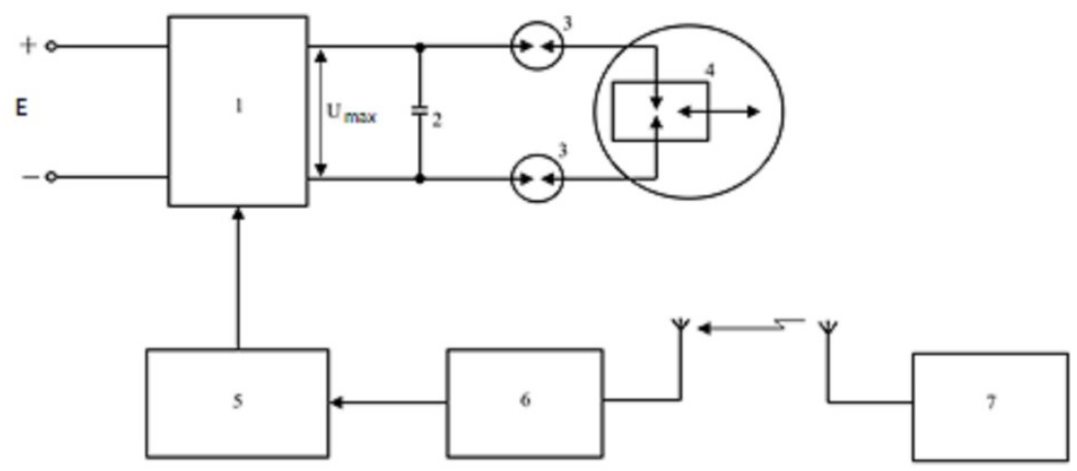

Fig. 3. Block scheme of the setup with shock excitation: 1 - step up voltage converter; 2 - storage capacitor; 3 - high-voltage dischargers; 4 - hydroacoustic radiator; 5 - control system; 6 - Bluetoothmodule; 7 - control signal transmitter. 
The voltage converter 1 is powered from an accumulator of $24 \mathrm{~V}$. The maximum voltage at the converter output $U_{\max }$ may reach $30 \mathrm{kV}$. The control system 5 , based on the microcontroller, allows us to adjust charge amplitude of the storage capacitor 2 and discharge pulse frequency. Two dischargers are applied in the discharge circuit. The second discharger is required to increase the discharge pulse steepness for the shock effect to be maximal. The hydroacoustic radiator with shock excitation 4 consists of a high-voltage discharger and an antenna. Two types of antennas were used as an antenna. They had the forms of parallel planes and of a cylinder.

The setup is controlled remotely by a Bluetooth-module 6 . A mobile phone is used as a control signal transmitter 7 . The setup control software allows us to adjust the discharge pulse amplitude and repetition frequency.

Physical configuration of the experimental setup is illustrated in Fig. 4. The left part (Fig. 4 , a) shows the electrohydrodynamic shock generator without a cover and the right part presents the cylindrical hydroacoustic radiator (Fig.4, b).

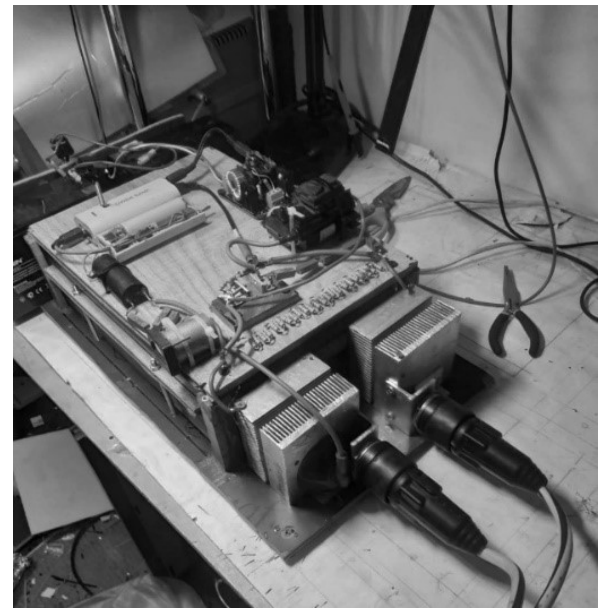

$a$

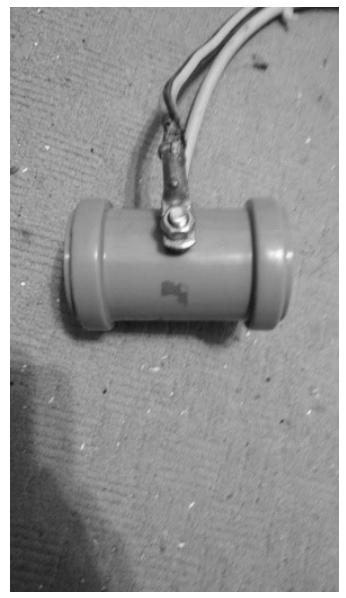

$b$

Fig. 4. Experimental equipment.

The investigations with the experimental setup were carried out in a pool of the depth of $1.5 \mathrm{~m}$ with the diameter of $3 \mathrm{~m}$. As a hydroacoustic signal sensor we used a hydrophone with piesoceramic primary transducer having the sensitivity of $140 \mu \mathrm{V} / \mathrm{Pa}$ and operating frequency range up to $20 \mathrm{kHz}$. To observe the recorded hydroacoustic signals, a digital edge-triggered oscilloscope produced by Keysight, model DSOX 1204A/G, was applied.

The hydroacoustic radiator and the hydrophone were installed at the distance of $2.45 \mathrm{~m}$ from each other. The radiator and the hydrophone were arranged so as to have enough time to receive the investigated signal before reflected signal arrival. The depth was $1 \mathrm{~m}$. The radiator was mounted on a rotator.

Fig. 5 shows the oscillogram of the received signal. The signals were received for the following operating mode of the setup: voltage on the storage capacitor was $7 \mathrm{kV}$, repetition frequency was $2 \mathrm{~Hz}$ and the radiator rotation angle was 45 decrease relatively the hydrophone. 


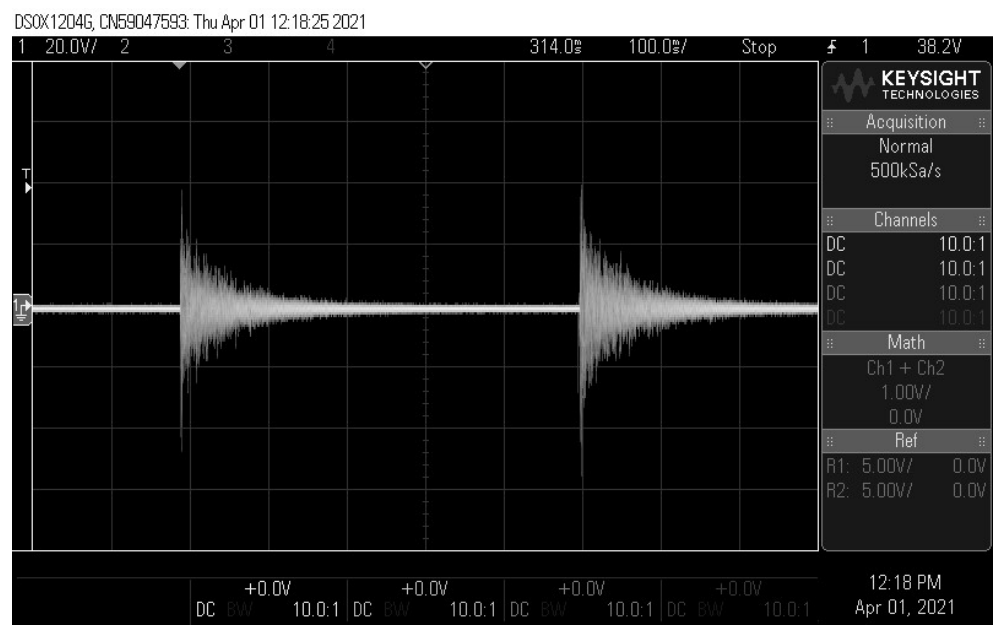

Fig. 5. Received signal oscillogram.

The measurement results for several rotation angles of the radiator are illustrated in Table 1.

Table 1. The measurement results for several rotation angles of the radiator

\begin{tabular}{|c|c|c|c|c|}
\hline $\begin{array}{c}\text { Radiator } \\
\text { rotation angle } \\
\text { relatively the } \\
\text { receiver, } \varphi\end{array}$ & \multicolumn{2}{|c|}{$\begin{array}{c}\text { Experimental } \\
\text { effective sound } \\
\text { pressure, Pexp }\end{array}$} & \multicolumn{2}{|c|}{$\begin{array}{c}\text { Estimated } \\
\text { effective sound } \\
\text { pressure, Pest }\end{array}$} \\
\cline { 2 - 5 } & $\mathrm{kPa}$ & p.u. & $\mathrm{kPa}$ & p.u. \\
\hline 0 degrees & 252 & 2,9 & 234 & 2,7 \\
\hline 45 degrees & 177 & 2,1 & 175 & 2,0 \\
\hline 90 degrees & 85,8 & 1 & 86,1 & 1 \\
\hline
\end{tabular}

\section{Experimental investigation of acoustic wave propagation in the presence of ice hummock}

An information-measuring system, illustrated in Fig. 6 [4] was used for the experimental investigation of hydroacoustic wave propagation along ice cover with hummocks.

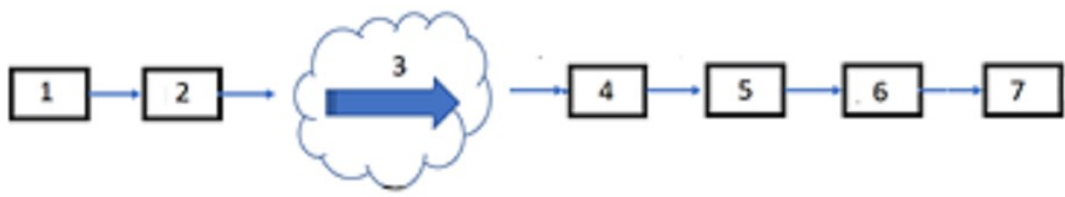

Fig. 6. Functional diagram of the information-measuring system: 1 - hydroacoustic setup with shock excitation (Fig. 4); 2 - hydroacoustic radiator; 3 - hydroacoustic wave propagation channel; 4 hydrophone; 5 - hydrophone amplifier; 6 - analogue-to-digital converter; 7 - PC.

Fig.7 shows the scheme of the hydroacoustic radiator and the hydrophone installation relatively the ice cover. The hydroacoustic radiator 2 is submerged into a hole, made in the ice, at the required depth. Hydrophone 4 is submerged into another hole at the required distance $x_{i}$ from the first one. 
A hydroacoustic pulse is sent by the hydroacoustic radiator 2, received by the hydrophone 4 , amplified by the hydrophone amplifier 5, converted into a digital code in the unit 6 and sent to a PC 7 for processing and information display.

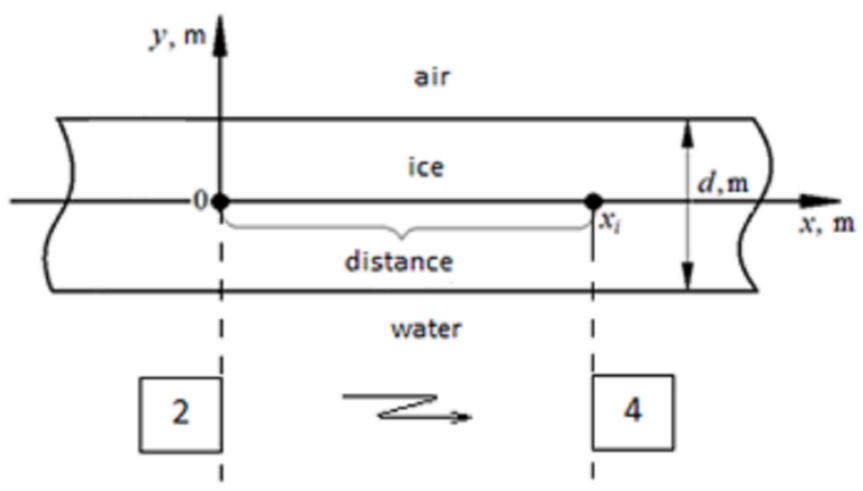

Fig.7. Scheme of radiator and receiver installation.

Fig. 8 shows the results for the experimental investigation of the dependence of sound pressure $(\mathrm{Pa})$, generated by cylindrical antenna in the direction of radiation axis direction, on the distance $(\mathrm{m})$. Five independent measurement series are presented.

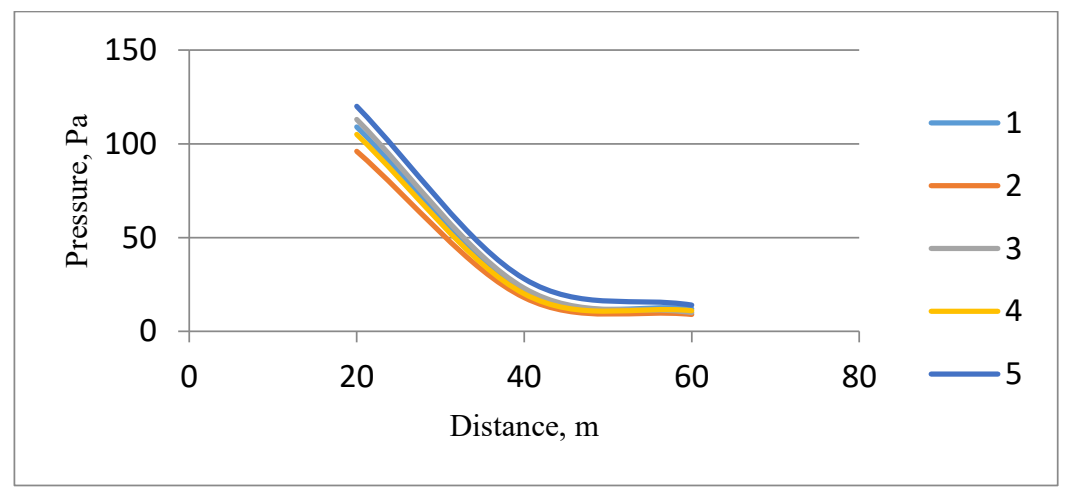

Fig.8. Curves of pressure dependence on distance.

\section{Conclusions}

The proposed mathematical model of elastic wave propagation may be applied for modeling of hydroacoustic wave propagation from earthquake sources under real ice conditions.

The experimental investigations confirmed high efficiency of the generator with electrohydraulic shock to form hydroacoustic high-power signals in the systems for signal transmission for extra-long distances. 


\section{References}

1. V.I. Korochentsev, Wave problems of the theory of directed and focus antennas: monography, (Dal'nauka, Vladivostok, 1998).

2. A.V. Korchaka, A.A. Em, V.I. Korochentsev, The Far Eastern Federal University: School of Engineering Bulletin, 38, 1 (2019) (in Russian).

3. V. Korochentsev, A. Em, Y. Shpak, W. Xue, G. Shabanov, E3S Web Of Conf., 127 (2019).

4. W. Chen, V. Chernenko, V. Petrosyants, V. Grishchenko, E3S Web Conf. 127 (2019). 